\title{
Clostridium novyi
}

National Cancer Institute

\section{Source}

National Cancer Institute. Clostridium novyi. NCI Thesaurus. Code C86285.

A species of obligately anaerobic, Gram positive, rod shaped bacteria assigned to the phylum Firmicutes. This species is endospore forming, hemolytic, requires the presence of thiols to grow in culture and produces a variety of toxins. C. novyi is pathogenic, causing gas gangrene within wounds and in individuals with diabetes. 